\title{
AUTOMORPHISMS OF SOLVABLE GROUPS
}

\author{
BY S. BACHMUTH AND H. Y. MOCHIZUKI ${ }^{1}$ \\ Communicated by Hyman Bass, October 15, 1974
}

We state an analogue of Tits' theorem on linear groups [3] as

Conjecture. Let $G$ be a finitely generated (f.g.) solvable group. Then, any f.g. subgroup of the automorphism group of $G$ is solvable by finite or contains a noncyclic free group.

As preliminary evidence, it was noticed by G. Baumslag and the authors that the Conjecture is correct when $G$ is nilpotent-by-abelian.

$Z G$ denotes the integral group ring of a group $G$ and $Q(D)$ the division ring of quotients of an Ore domain $D$. If $Z G$ is an Ore domain and $U$ a group of matrices over $Q(Z G)$, we say $U$ has a (right) common denominator if there is $b \in Z G$ such that each entry in a matrix of $U$ has the form $a b^{-1}, a \in Z G$.

Henceforth these notations hold. $F$ a free group whose rank will be specified, $R \neq\{1\}$ a normal subgroup of $F, \gamma_{n}(R)$ the $n$th term of the lower central series of $R, R^{\prime}=\gamma_{2}(R), H=F / R, G=F / R^{\prime}, A(G)$ the automorphism group of a group $G, A\left(G_{1} ; G_{2}\right)$ the kernel of $A\left(G_{1}\right) \rightarrow A\left(G_{2}\right)$.

Theorem 1 is joint work with E. Formanek.

THEOREM 1. Let $F$ have rank two and assume $Z(F / R)$ is a domain with $R \leqslant F^{\prime}$. Then $A\left(F / R^{\prime} ; F / F^{\prime}\right)$ consists entirely of inner automorphisms.

COROllary. Let $F / R$ be as in Theorem 1 and also assume $F / R$ is solvable. Then $F / \gamma_{n}(R)$ satisfies the Conjecture.

Problem 1. Let $F$ have rank two and $H=F / R$ be a solvable group such that $Z H$ is an Ore domain, and let $U$ be a group of units of $Q(Z H)$ which has a common denominator. Is $U$ a solvable group?

An affirmative answer to Problem 1 would yield a proof of the Corollary independent of Theorem 1. It seems reasonable to suspect that a group $U$ of units in $Q(\mathrm{ZH}), \mathrm{ZH}$ an Ore domain, having a common denominator is in fact a conjugate of a group of units of $Z H$.

AMS (MOS) subject classifications (1970). Primary 20F55, 20E99; Secondary $20 \mathrm{H} 25$.

1 Both authors were supported by NSF Grant No. GP-38968.

Copyright $\odot 1975$, American Mathematical Society 
THEOREM 2. Let $\mathrm{H}$ be a poly-(infinite cyclic) group, and let $U$ be a f.g. subgroup of $\mathrm{SL}_{2}(Q(Z H))$ which has a common denominator. Then, $U$ contains a noncyclic free subgroup, or $U$ is a finite extension of a radical group.

A radical group has an ascending series terminating in the group such that each factor group of the series is locally nilpotent. As a corollary of Theorem 2, we have

THEOREM 3. Let $F$ have rank three and $H=F / R$ be poly-(infinite cyclic). Let $A$ be a f.g. subgroup of $A(G ; H)$. (i) If A satisfies the maximum condition on abelian subgroups, then $A$ contains a noncyclic free subgroup or $A$ is polycyclic by finite. (ii) If A satisfies the minimum condition on abelian subgroups, then $A$ is a Černikov group (i.e., abelian by finite satisfying the minimum condition on subgroups). (iii) If each normal subgroup of $A$ is $f . g$. , then A contains a noncyclic free subgroup or $A$ is polycyclic by finite. Moreover, (i)-(iii) are true if $A$ is a f.g. subgroup of $A\left(F / \gamma_{n}(R)\right)$.

Problem 2. Can one improve Theorem 3 to conclude that $A$ contains a noncyclic free group or is solvable-by-finite without restrictions on $A$ ?

A positive answer to Problem 2 would follow if one could show that a locally solvable subgroup of $\mathrm{SL}_{2}(Q(Z H))$ is solvable; e.g., by showing that there is a bound on the derived length of a solvable subgroup of $\mathrm{SL}_{2}(Q(Z \mathrm{H}))$. There is one nice case in which we can answer Problem 2.

THEOREM 4. Let $F$ have rank three and let $F / R$ be torsion-free nilpotent of class two. Then, $F / \gamma_{n}(R)$ satisfies the Conjecture.

Problem 3. Let $G$ be a solvable group such that $Z G$ is an Ore domain. If $U$ is a f.g. subgroup of $\mathrm{SL}_{2}(Q(Z G))$ which has a common denominator, what conclusion can one draw concerning $U$ ?

THEOREM 5. Let $F$ have rank $n$, and $H=F / R$ be free metabelian. Then, any f.g. subgroup of $\mathrm{SL}_{2}(\mathrm{ZH})$ is solvable-by-finite or contains a noncyclic free subgroup.

Our concluding result adds to the evidence for the Conjecture by indicating the prevalence of free subgroups.

THEOREM 6. Let $F$ have rank three and $H=F / R$ be any group for which $Z(H)$ has no nonzero zero divisiors. Let $A_{i}(1 \leqslant i \leqslant 3)$ be the (abelian) 
subgroup of $A(G ; H)$ whose nonidentity elements leave all but the ith generator fixed. Then, the subgroup of $A(G ; H)$ generated by the $A_{i}$ is the free product of the $A_{i}$.

The restriction on the rank of $F$ is not essential. A similar more complicated result holds for $F$ of rank $>3$.

The pioneering work of Magnus described the relevant automorphism groups as automorphisms of free modules in an explicit way. The work announced here is a start in exploiting Magnus' representations in noncommutative contexts. In our proofs we relied on the structure of a skew-polynomial domain $K[x], K$ a division ring, and its division ring of quotients $Q(K[x])$ with the induced discrete valuation. The decomposition of $\mathrm{SL}_{2}(Q(K[x]))$ and $\mathrm{SL}_{2}(K[x])$ as amalgamated free products of groups due to Ihara and Nagao, respectively [2], were employed, and also the subgroup theorems for amalgamated products of Karrass and Solitar [1].

We conclude with a more specific conjecture which has a deeper relationship with Tits' theorem. Henceforth a linear group will mean a group of matrices over a commutative Noetherian ring. We call a group poly $L$ if the group has a finite subnormal series such that the factors are either linear or abelian groups. Our suggestion is that

"The group of automorphisms of a f.g. solvable group is poly-L."

F.g. nilpotent-by-abelian groups have automorphism groups which are poly- $L$ (this includes all polycyclic groups). Theorem 1 tells us that the automorphism groups of a large class of 2-generator nilpotent-by-torsion-free solvable groups are poly- $L$.

ACKNOWLEDGEMENT. We thank G. Baumslag for his interest.

\section{REFERENCES}

1. A. Karrass and D. Solitar, The subgroups of a free product of two groups with an amalgamated subgroup, Trans. Amer. Math. Soc. 150 (1970), 227-255. MR 41 \#5499.

2. J.-P. Serre with collaboration of H. Bass, Groupes discrets, Collége de France Notes, $1968-1969$.

3. J. Tits, Free subgroups in linear groups, J. Algebra 20 (1972), 250-270. MR 44 \#4105.

DEPARTMENT OF MATHEMATICS, UNIVERSITY OF CALIFORNIA, SANTA BARBARA, CALIFORNIA 93106 